\title{
Dermatology
}

Dermatology 2015;230:128-134

DOI: $10.1159 / 000368348$
Received: November 29, 2013

Accepted after revision: September 2, 2014

Published online: February 3, 2015

\section{1,064-nm Diode Laser Therapy of Onychomycosis: Results of a Prospective Open Treatment of 82 Toenails}

\author{
Regina Renner Kathrin Grüßer Michael Sticherling \\ Hautklinik Erlangen, Universitätsklinikum Erlangen, Erlangen, Germany
}

\section{Key Words}

Onychomycosis - Diode laser therapy · Tinea unguis ·

Treatment

\begin{abstract}
Background: Onychomycosis is one of the most prevalent nail disorders in adults, where conventional topical therapy is often protracted and in most cases ineffective. On the other hand, systemic/oral therapy is not suitable for all patients and might be associated with relevant side effects. Therefore, laser therapy can be used as an alternative to or extension of existing treatment protocols. Objectives: We used diode laser treatment for onychomycosis to evaluate the efficacy of this treatment as single therapy as well as in combination with an ongoing antimycotic treatment. Methods: We used a 1,064-nm diode laser (FOX Laser, ARC Laser GmbH, Germany) in pulse mode with a spot size of $4 \mathrm{~mm}$. The laser's settings were: $8 \mathrm{~W}$, with a pulse duration of $80 \mathrm{~ms}$ and a repetition rate of $5.6 \mathrm{~Hz}$; the energy fluence/pulse was $5.1 \mathrm{~J} / \mathrm{cm}^{2}$, and a total energy of 250-500 J for large and/or thickened nails (digit I), 120-200 J for medium nails (digits II-IV) and 60-120 J for small and/or very thin nail plates (digit V) was counted in the laser display. Two to three passes where applied continuously over the entire area of the nail (nail plate, nail folds and eponychium) in a grid pattern. Eighty-two af-
\end{abstract}

fected toenails were treated at least twice every 8 weeks; all nails treated were clinically diagnosed as onychomycosis. The evaluation of the treated nails was conducted by two dermatologists, initially by semiquantitative visual examination, followed by evaluation with the Onychomycosis Severity Index (OSI). In addition, the patients were asked to state their satisfaction with the treatment and its results in a written questionnaire. Results: All nails showed an improvement ranging between 14 and 56\% including the analytical evaluation scale (OSI). Two dermatologists evaluated 34 nails. Both reported significant improvement and/or good clinical improvement, which corresponds to about $41 \%$ for both evaluations. The mean OSI of all patients was 18.9/19.9 before treatment which changed to $14.3 / 14.8$ after treatment. This corresponds to an improvement of about $25 \%$ $(-4.6 /-5.1)$ compared to the initial value. Specifically the subgroup with positive antimycotic culture and no additional antimycotic treatment showed an improvement of about $25 \%$. This improvement can be attributed to the effect of the diode laser treatment. When evaluating the OSI of all nails, it appears that about $15-20 \%$ of the severe OSIs improved and changed to a moderate level, while a similar portion of patients with moderate onychomycosis improved to mild onychomycosis. Patient satisfaction was measured to an average value of 4.6 out of a maximum of 10 points. About $60 \%$ of the treated patients would recommend the treatment to

\section{KARGER 125}

(c) 2015 S. Karger AG, Base

$1018-8665 / 15 / 2302-0128 \$ 39.50 / 0$ 
their family members or friends. Conclusion: This investigation demonstrated that diode laser treatment of onychomycosis provides acceptable results with minimal to no side effects. Further clinical evaluations could help to establish better therapy protocols, especially for those patients who had no benefit from the laser treatment, or could also be used as an add-on to an existing therapy.

(c) 2015 S. Karger AG, Basel

\section{Introduction}

Onychomycosis is the most widespread nail disease in adults around the world [1] with about $10 \%$ of the German population affected. In most cases, Trichophyton rubrum [1] is involved. Unfortunately, the application of topical and systemic antimycotic drugs is tedious and associated with a significant number of relapses [2]. Especially for patients with pre-existing diseases as well as for pregnant and breastfeeding female patients, reliable alternatives to systemic treatments are lacking.

In 2010, the US Food and Drug Administration approved different laser systems for the so-called 'temporary increase in clear nails' in patients with onychomycosis. Fungal eradication might be mediated by heat in infrared laser systems or by the disruption of fungi and spores by laser pulses e.g. in Q-switched lasers. Lasers seem to be an ideal treatment for onychomycosis due to their minimally invasive nature as well as their high potential to restore clear nail growth with just a few treatment sessions. For onychomycosis, various laser systems including carbon dioxide, neodymium-doped yttrium aluminium garnet, an 870/930-nm combination, and femtosecond infrared 800-nm lasers are available, in addition to photodynamic and ultraviolet light therapy. Protocols for a safe and effective treatment, either alone or in combination with conventional treatment options, are currently investigated. Based on these ideas, we evaluated in this prospective open treatment the effect of a new and easy-to-handle 1,064-nm diode laser as a single therapeutic option and as an add-on therapy to an already ongoing but insufficient antimycotic therapy.

\section{Patients and Methods}

\section{Patients}

Inclusion criteria were patients older than 18 years with typical clinical onychomycotic changes of their toenails or patients who had already been treated with antimycotics for at least 8 weeks without improvement. Types of onychomycosis conditions were:
Table 1. Mean values of the total energy applied in joules per whole nail

\begin{tabular}{llllll}
\hline & Digit I & Digit II & Digit III & Digit IV & Digit V \\
\hline 1st treatment & 369 & 165 & 177 & 178 & 152 \\
2nd treatment & 425 & 245 & 140 & 140 & 172 \\
\hline
\end{tabular}

total dystrophic onychomycosis, distolateral subungual onychomycosis, proximal subungual onychomycosis with and without paronychia and superficial white onychomycosis.

Exclusion criteria were patients with coloured nail polish, patients with nail plates exhibiting an unnatural colour change due to bacterial infections or other topical treatments; patients treated with drugs that might cause excessive light sensitivity; patients with ongoing systemic antibiotic treatment within the last 6 months; patients going through systemic treatment with corticosteroids, antidepressants or anticoagulants; patients who were pregnant; patients with nails having subungual pigmentation caused by haematoma or naevus; patients with other clinical nail diseases, such as psoriasis, lichen planus or atopic dermatitis; patients with peripheral neuropathy and patients with an acute inflammation of their toes or nails. Native and fungal cultures were performed for the affected nails before initiation of the diode laser treatment to verify the presence of onychomycosis causing pathogens.

Observing these criteria, 24 patients were included. Although the ARC Laser device is an approved medical device for treating onychomycosis ('temporary increase in clear nails') both in Europe and Canada ('Health Canada Approved'), all patients were asked to sign an informed consent form, including a data processing form, before starting the treatment and were informed about the risks and clinical procedures of laser treatment and alternative procedures to laser treatment.

Eight patients were treated 3 times because of the extent of their disease involving the entire nail plate with excessive thickness and involvement of the nail matrix; all other patients were treated twice at intervals of 8 weeks. One female patient, who only showed up for her initial treatment, was excluded from data evaluation. In total, 82 toenails of 23 included patients could be evaluated.

The patient distribution was: 13 males (mean age 62 years, median 62 years, min. 33 years, max. 84 years) and 10 females (mean age 53 years, median 58 years, min. 26 years, max. 84 years). In 13 patients $(57 \%)$, the native or fungal culture was positive with T. rubrum in 8 cases, moulds in 4 cases and yeasts in 1 case. For patients with positive mould culture, none could be found to be associated with dermatophyte infection.

Fifteen patients had already used topical antimycotic treatment (ciclopiroxolamine, amorolfine or clotrimazole) for $>8$ weeks, with 7 of these patients still showing positive native or cultural fungus results at the beginning of our treatment. In 2 of 3 patients on systemic terbinafine $250 \mathrm{mg} /$ week, mould cultures were also still positive. However, all 15 patients reported no improvement despite their antimycotic therapy of $>8$ weeks. Therefore, in these patients, laser treatment was used in addition to ongoing antimycotic treatment. 
Table 2. Evaluation of the OSI score in the different groups before/after treatment by the two dermatologists

\begin{tabular}{lll}
\hline & Dermatologist 1 & Dermatologist 2 \\
& & \\
\hline Group 1 (all patients) & $18.9 / 14.3(25)$ & $19.9 / 14.8(25)$ \\
Group 2 (positive fungal culture) & $19.7 / 16.2(18)$ & $19.5 / 12.6(35)$ \\
Group 3 (negative fungal culture) & $17.8 / 11.5(35)$ & $20.1 / 16.4(18)$ \\
Group 4 (without antimycotic treatment) & $18.8 / 14.0(25)$ & $19.6 / 13.7(25)$ \\
Group 5 (with antimycotic treatment) & $19.0 / 14.4(25)$ & $20.0 / 15.3(25)$ \\
Group 6 (positive fungal culture but no antimycotic treatment) & $17.5 / 13.2(25)$ & $17.8 / 12.9(28)$ \\
Group 7 (positive fungal culture and antimycotic treatment) & $21.7 / 18.7(14)$ & $22.1 / 19.3(13)$ \\
Group 8 (moulds) & $17.7 / 7.6(56)$ & $17.7 / 7.4(58)$ \\
\hline
\end{tabular}

Results are expressed as values before/after treatment with the percent improvement in parentheses.

After completion of the diode laser treatment and final photographic documentation, all patients received anonymous evaluation forms, of which 22 of 23 were completed.

Methods

The FOX is a diode laser (ARC Laser GmbH, Germany) with a wavelength of $1,064 \mathrm{~nm}$ in a gated laser mode and a spot size of $4 \mathrm{~mm}$. The laser default settings suggested by the manufacturer's instructions were $8 \mathrm{~W}, 80 \mathrm{~ms}$ pulse duration, a repetition rate of $5.6 \mathrm{~Hz}$, which results in an energy fluence/pulse count of $5.1 \mathrm{~J} / \mathrm{cm}^{2}$, and a total energy of 250-500 J for large and/or thickened nails (digit I), 120-200 J for medium nails (digits II-IV) and 60-120 J for small and/or very thin nail plates (digit V) counted in the laser display. The mean values of total energy in joules applied to the patients' whole nail are shown in table 1 . Through the patients' thermal feedback, the investigator made sure that the treatment was applied correctly. Thicker nails were debrided and 2-3 laser passes/nail were normally applied.

The laser beam was applied continuously with an average swipe speed of around $2 \mathrm{~mm} / \mathrm{s}$ over the entire area of the nail (nail plate, nail fold and eponychium) in a grid pattern. The treatment was stopped intermittently if the patient reported discomfort due to the heat caused by the laser and was continued until the patient reported the same discomfort. However, the temperature increase was very mild with this diode laser technology and its gated laser mode. The treatment was stopped for this session once the target energy was reached. After the treatment, patients were asked to wear new socks and to continue the ongoing treatment, if there was any.

The patients were re-evaluated 3.2 months (mean value) after their first treatment (min. 1.8 months, max. 7.2 months). The final evaluation using photographs was performed semiquantitatively by 2 dermatologists via a visual assessment of the nail and visible improvement, followed by an evaluation with the Onychomycosis Severity Index (OSI). The OSI is a simple, objective and reproducible numeric classification system for grading the severity of onychomycosis [3]. The score is obtained by multiplying the score for the area of involvement (range $0-5$ points) by the score for the proximity of the disease to the matrix (range $1-5$ points). Additional 10 points were added when longitudinal streaks of more than $2 \mathrm{~mm}$ were present for subungual hyperkeratosis. Mild onychomycosis corresponds to scores of 1-5, moderate to 6-15 and severe onychomycosis to $16-35$ points.

\section{Results}

To evaluate the results, all pictures before and after treatment were evaluated separately by a second dermatologist who was completely independent of this investigation. An overview of the OSI scores is shown in table 2.

Furthermore, the patients evaluated their expectations and assessment of their therapeutic success in an anonymous questionnaire. As this diode laser treatment and a number of other standard antimycotic treatments, e.g. nail polish, are not covered by German health insurance companies, patients were asked how much they had already invested for their onychomycosis treatment and how much they would have been willing to pay for a diode laser treatment.

\section{Semiquantitative Clinical Evaluation of the Laser Treatment by Dermatologist No. 1}

In total, 82 nails were treated. Thereof, 14 nails (17.1\%) of 6 patients showed significant clinical improvement (example in fig. 1), and 19 nails (23.2\%) of 14 patients showed good clinical improvement (example in fig. 2). Of these 33 improved nails (40.3\%), 6 patients were diagnosed as negative in the native fungal examination and/or culture. Forty-nine nails (59.8\%) showed no changes between their first and last photographic documentation. One patient developed a slight redness of the nail folds at both toenails of digits I about 14 days after his first laser treatment, without further symptoms. No other significant side effects were reported or observed in any other patient. 

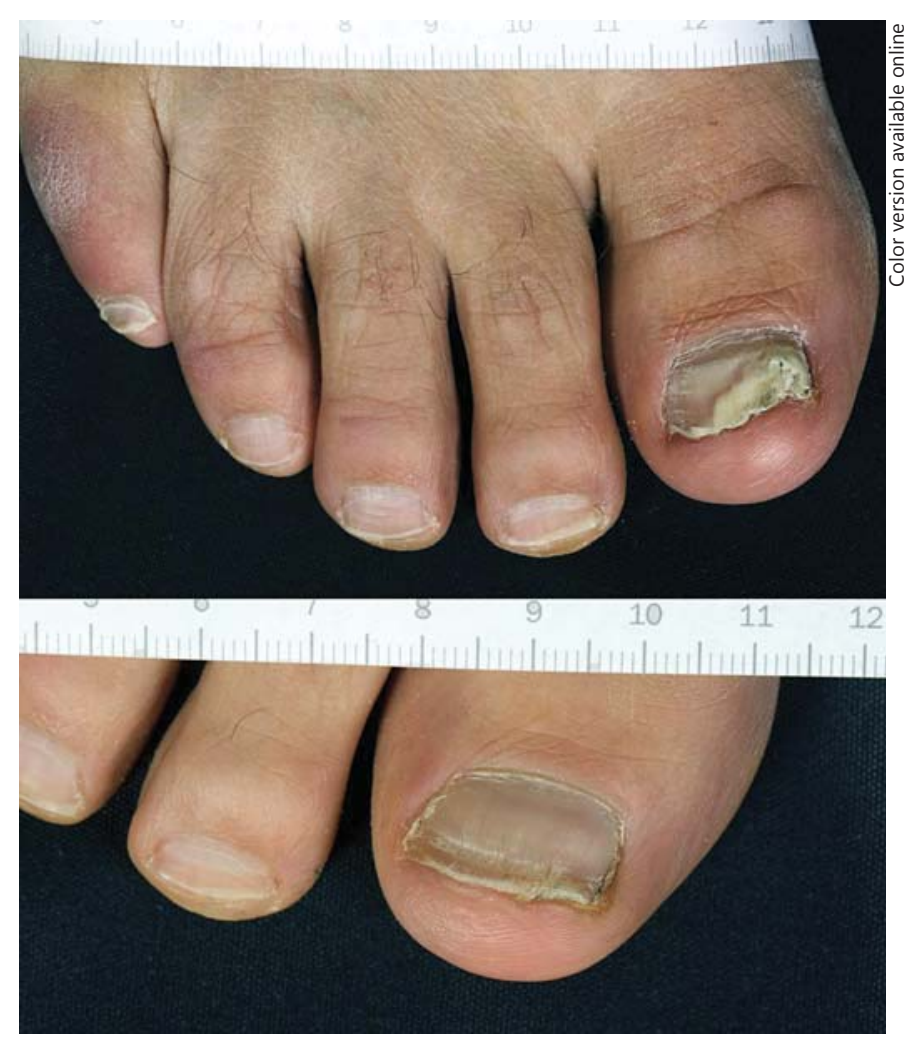

Fig. 1. An example of distinct clinical improvement.

Semiquantitative Clinical Evaluation of the Laser

Treatment by Dermatologist No. 2

Twelve (14.6\%) of 82 nails in 7 patients were evaluated as significantly improved, and 22 nails (26.8\%) of 14 patients with good clinical improvement. In total, 34 (41.4\%) of the treated nails showed clinical improvement. Fortyeight nails (58.5\%) showed no changes between their first and last photographic documentations. No significant side effects were reported or observed.

\section{Evaluation via the OSI (Dermatologist No. 1/}

Dermatologist No. 2)

Group 1: All Patients $(n=23)$. The mean initial OSI of all patients was 18.9/19.9 and showed improvement after laser treatment to $14.3 / 14.8$ (mean value), which is a change of about $25 \%(-4.6 /-5.1$ points) compared to the initial value.

Group 2: Patients with Positive Fungal Culture $(n=13)$. Patients with positive fungal culture showed an initial OSI of 19.7/19.5 (mean value) with improvement after laser treatment to $16.2 / 12.6$ (mean value), which means a

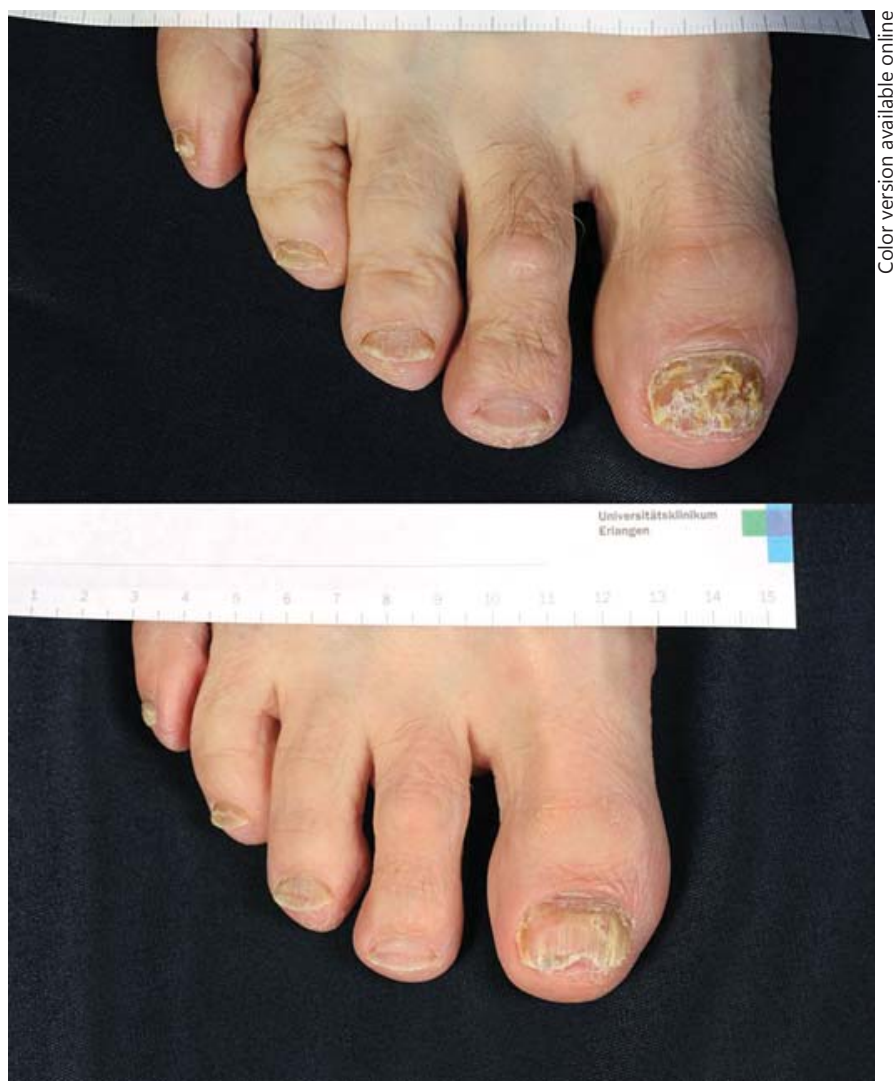

Fig. 2. An example of good clinical improvement.

change of about $18 \% / 35 \%$ ( $-3.5 /-6.9$ points) compared to the initial value.

Group 3: Patients with Negative Fungal Culture ( $n=$ 10). Patients with negative fungal culture showed an initial OSI of 17.8/20.1 (mean value) with improvement after laser treatment to $11.5 / 16.4$ (mean value). This means a change of about $35 \% / 18 \%(-6.3 /-3.7$ points) compared to the initial value.

Group 4: Patients without Antimycotic Treatment ( $n=$ 8). Patients without any prior or ongoing antimycotic treatment showed an initial OSI of 18.8/19.6 (mean value) with improvement after laser treatment to $14.0 / 13.7$ (mean value), which means a change of about $25 \%(-4.8 /$ -5.9 points) compared to the initial value.

Group 5: Patients with Ongoing Antimycotic Treatment $(n=15)$. Patients with antimycotic treatment showed an initial OSI of 19.0/20.0 (mean value) with improvement after laser treatment to $14.4 / 15.3$ (mean value). This corresponds to a change of about $25 \%(-4.6 /-4.7$ points) compared to the initial value. 
Group 6: Patients with Positive Fungal Culture but without Antimycotic Treatment $(n=7)$. Patients with positive fungal culture but without antimycotic treatment showed an initial OSI of 17.5/17.8 (mean value) with improvement after laser treatment to $13.2 / 12.9$ (mean value) points. This means a change of about $25 \% / 28 \%(-4.3 /-4.9$ points) compared to the initial value.

Group 7: Patients with Positive Fungal Culture and Ongoing Antimycotic Treatment $(n=6)$. Patients with positive fungal culture and with additional antimycotic treatment showed an initial OSI of 21.7/22.1 (mean value) with improvement after laser treatment to 18.7/19.3 $(-3.0 / 2.8$ points, mean value). This means a change of about $14 \% / 13 \%$ compared to the initial value. This is the group with the worst OSI at the beginning of our treatment and the smallest improvement.

Group 8: Patients with Moulds $(n=4)$. Patients with moulds only in fungal culture showed an initial OSI of 17.7/17.7 (mean value) with improvement after laser treatment to 7.6/7.4 (mean value). This means a change of about $56 \% / 58 \%$ ( $-10.1 / 10.3$ points) compared to the initial value.

\section{Patient Questionnaire}

The questionnaire (table 3) was answered by 22 patients (95\%, mean age 58 years). Patients had great expectations regarding the treatment results at the beginning of the therapy [mean value $7.5(0-10)]$. These expectations were not met in all patients [mean value $4.4(0-10)$ ].

Thirteen patients declined to pay for the laser treatment. The remaining 9 patients mentioned that they would have paid about EUR 56 (mean value). In total, all patients had already invested EUR 126 for their onychomycosis treatment before their consultation in the clinic.

Fourteen patients (58\%) would recommend the treatment to a friend or family member. In 6 patients, selfesteem was improved after the treatment. Satisfaction in total with the treatment was 4.6 points $(0-10)$. Two patients mentioned a reduced growth of their nails as a possible side effect of the treatment (which could not be objectified), and 1 patient mentioned pain (but without any time context documented).

\section{Discussion}

All treated patients showed a high OSI (mean value) at the initiation of the treatment, and all patients improved after the treatment. This was obvious from the semiquantitative clinical evaluation done by the 2 dermatologists as
Table 3. Results of the patient questionnaire

\begin{tabular}{|c|c|c|c|c|c|}
\hline $\begin{array}{l}\text { Age, } \\
\text { years }\end{array}$ & Sex & $\begin{array}{l}\text { Patients' } \\
\text { expectations } \\
(0-10)\end{array}$ & $\begin{array}{l}\text { Expecta- } \\
\text { tions met? } \\
(0-10)\end{array}$ & $\begin{array}{l}\text { Side } \\
\text { effects? }\end{array}$ & $\begin{array}{l}\text { Satisfaction } \\
\text { in total } \\
(0-10)\end{array}$ \\
\hline 67 & $\mathrm{w}$ & 10 & 0 & no & 0 \\
\hline 33 & $\mathrm{~m}$ & 8 & 0 & no & 0 \\
\hline 82 & $\mathrm{~m}$ & 8 & 2 & RNG & 2 \\
\hline 62 & n.a. & 7 & 5 & no & 8 \\
\hline 69 & $\mathrm{~m}$ & 10 & 10 & no & 10 \\
\hline 27 & $\mathrm{w}$ & 7 & 0 & no & 0 \\
\hline 47 & $\mathrm{~m}$ & 8 & 0 & no & 0 \\
\hline 50 & $\mathrm{w}$ & 8 & 7 & no & 7 \\
\hline 30 & $\mathrm{w}$ & 5 & 5 & RNG & 5 \\
\hline 57 & $\mathrm{~m}$ & 7 & 0 & no & 0 \\
\hline 55 & $\mathrm{~m}$ & 6 & 0 & no & 0 \\
\hline 62 & $\mathrm{~m}$ & 9 & 6 & no & 6 \\
\hline 84 & $\mathrm{~m}$ & 8 & 2 & no & 2 \\
\hline 54 & $\mathrm{~m}$ & 5 & 5 & no & 3 \\
\hline 79 & $\mathrm{~m}$ & 5 & 7 & no & 7 \\
\hline 39 & $\mathrm{w}$ & 7 & 5 & no & 7 \\
\hline 62 & $\mathrm{w}$ & 10 & 10 & no & 10 \\
\hline 67 & $\mathrm{~m}$ & 9 & 8 & no & 7 \\
\hline 76 & $\mathrm{~m}$ & 7 & 7 & no & 8 \\
\hline 48 & $\mathrm{~m}$ & 8 & 8 & no & 9 \\
\hline 76 & $\mathrm{w}$ & 6 & 6 & no & 5 \\
\hline 55 & $\mathrm{w}$ & 7.5 & 1.5 & pain & 1.5 \\
\hline mean 58 & - & mean 7.5 & mean 4.4 & - & 4.6 \\
\hline
\end{tabular}

n.a. = No data available; $\mathrm{RNG}=$ retarded nail growth

well as from the evaluation via the OSI. The semiquantitative analysis showed an improvement of about $41 \%$ in all nails. The OSI improved absolutely by about 4 points in all patients as well as in all evaluated subgroups.

In a recent study performed with a 1,064-nm Nd:YAG laser system [4], the authors reported results similar to the current protocol with a reduction of about $4-5$ points in the OSI after the laser treatment. In our evaluation, patients with positive fungal culture, but without any other antimycotic treatment (group 6), showed an improvement after treatment of about $25 \%$ ( -4.3 points). This might be attributed to the laser treatment alone.

It seems curious at first that those patients with negative fungal culture and those only with moulds in their fungal culture improved, too. However, in these patients this might be due to the low amounts of fungi and spores, too low to give positive culture results. In contrast, patients with a high OSI and large amounts of fungi in nail plates are more difficult to treat. In our study, they improved by only $14 \%$ (-3 points) compared to their initial value. 
Table 4. Changes of the OSI according to its grading of mild, moderate and severe

\begin{tabular}{llll}
\hline Classification & $\begin{array}{l}\text { Mild OSI } \\
(1-5)\end{array}$ & $\begin{array}{l}\text { Moderate OSI } \\
(6-15)\end{array}$ & $\begin{array}{l}\text { Severe OSI } \\
(16-35)\end{array}$ \\
\hline Before treatment & $13 \%$ & $22 \%$ & $65 \%$ \\
After treatment & $29 \%$ & $25 \%$ & $46 \%$ \\
Changes per group & $16 \%$ & $-3 \%$ & $19 \%$ \\
\hline
\end{tabular}

It seems that about $15-20 \%$ of all severe cases improve and change to the moderate level, while a similar portion of patients with moderate onychomycosis improve to mild onychomycosis.

If we do not evaluate the OSI of single groups but the OSI of all nails, it seems that about $15-20 \%$ of all severe OSIs improve and change to the moderate level, while a similar portion of patients with moderate onychomycosis improve to mild onychomycosis (table 4).

Unfortunately, the patients' feedback is rather disappointing, especially regarding their satisfaction with the treatment results. This could be due to unrealistically high expectations regarding the laser technique, or it could be due to previous unsuccessful therapy or slow nail growth. The reluctance to pay for such treatment might be due to the fact that most patients had already invested in unsuccessful treatments before.

Other in vitro as well as clinical evaluations with a 1,064-nm Nd:YAG laser demonstrated divergent results [5-7], e.g. the study by Zhang et al. [6] where treatment was performed with a long-pulse Nd:YAG 1,064-nm laser using the following parameters: $240-324 \mathrm{~J} / \mathrm{cm}^{2}$ fluence, $30 \mathrm{~ms}$ pulse duration, $3 \mathrm{~mm}$ spot size and $1 \mathrm{~Hz}$ frequency. The laser energy was adjusted to the thickness of the nail plate. In 1 session, 3 passes across each nail plate with 2 -min pauses between passes were applied. A full course of treatment consisted of 4 sessions performed on days 0 , 7, 14 and 21. Group 1 received 2 courses of treatment (8 sessions), while group 2 received 1 single course (4 sessions). In group 1 , the effectivity rates at 8 and 16 weeks were 63 and 62,68 and $67 \%$ for group 2, without significance between any subgroup pair $(p>0.05)$. However, the variety of available systems and the advised parameters hamper a comparison of these results.

The efficacy of the laser therapy is most likely due to its thermal effect because the fungus mycelium is relatively heat sensitive [6]. In a study of Paasch et al. [8], the highest increase in temperature in a 980 -nm laser was found with a pulse of $6 \mathrm{~ms}$ and a fluence of $27 \mathrm{~J} / \mathrm{cm}^{2}$, with an inhibition of growth for Candida guilliermondii and T. interdigitale

Evaluation of a 1,064-nm Diode Laser

Therapy of Onychomycosis only at temperatures $>50^{\circ} \mathrm{C}$. All other pathogenic germs showed reduced, but not inhibited growth, and C. albicans showed no impact at all. This temperature dependency might be a reason for a possible failure of laser treatments, especially if nail growth is very slow (possibly due to the patient's age), and e.g. in our case, a subjective thermal feedback by the patient is needed, and frequently found pathogenic fungi like T. rubrum might only be reduced in growth but not completely eradicated. Possibly, a more repetitive application over time could be helpful.

In conclusion, a prolonged observation time might be necessary for a better clinical outcome but does not seem feasible under real life conditions. As seen in our patient questionnaire, patients have great expectations regarding their treatment. However, with only slow clinical improvement after 8-12 weeks, patients might change their nail treatment themselves due to their impatience. Recent studies indicate a longer treatment period and a prolonged follow-up period of at least 3-6 months [4].

On the other hand, topical antifungal treatments show high failure rates of $61-64 \%$ for e.g. ciclopiroxolamine after 48 weeks. This might be due to a lack of compliance because of repetitive daily applications over months [9]. The number of treatment sessions might be the great advantage of the laser treatment; they are short, and the dermatologist can ensure the treatment is performed accurately.

\section{Limitations}

Limitations of this evaluation to be pointed out are the short observation period as well as the fact that patients with negative fungal results, though clinically highly suggestive of onychomycosis, were included.

\section{Conclusion}

This evaluated treatment demonstrates that diode laser as a therapy for onychomycosis provides acceptable improvements between 14 and 56\%, yet causes minimal to no side effects; this could be shown in the semiquantitative visual clinical evaluation as well as in the detailed evaluation via the OSI.

In the subgroup with patients with positive antimycotic culture, but without additional antimycotic treatment, an improvement was demonstrated in about $25 \%$ of all cases, which seems to be due to the effect of the diode laser treatment. There might be subgroups that benefit more from the treatment. When evaluating the total OSI of the nails, it seems that about $15-20 \%$ of all severe OSIs 
improve and change to the moderate level, while a similar portion of patients with moderate onychomycosis improve to mild onychomycosis.

Further clinical evaluations should help to establish better therapy protocols, especially for those patients who did not benefit enough from the current laser treatment. Such evaluations may also assist in even further improving the results for those patients who responded well to the treatment and may also be used as an add-on to an existing therapy.

\section{Acknowledgements}

We would like to thank Ms. Stephanie Friedel for her help in scheduling the patients and for her diligent documentation, and Mr. Marcel Ernstberger from the ARC Laser Company for his helpful suggestions to improve the paper.

\section{Disclosure Statement}

ARC Laser GmbH provided the corresponding author with a FOX diode laser for 6 months to evaluate its efficacy.

\section{References}

1 Ghannoum MA, Hajjeh RA, Scher R, Konnikov N, Gupta AK, Summerbell R, Sullivan S, Daniel R, Krusinski P, Fleckman P, Rich P, Odom R, Aly R, Pariser D, Zaiac M, Rebell G, Lesher J, Gerlach B, Ponce-De-Leon GF, Ghannoum A, Warner J, Isham N, Elewski B: A large-scale North American study of fungal isolates from nails: the frequency of onychomycosis, fungal distribution, and antifungal susceptibility patterns. J Am Acad Dermatol 2000;43:641-648.

2 Finch JJ, Warshaw EM: Toenail onychomycosis: current and future treatment options. Dermatol Ther 2007;20:31-46.
3 Carney C, Tosti A, Daniel R, Scher R, Rich P, DeCoster J, Elewski B: A new classification system for grading the severity of onychomycosis: Onychomycosis Severity Index. Arch Dermatol 2011;147:1277-1282.

4 Hees H, Jäger MW, Raulin C: Treatment of onychomycosis using the 1,064-nm Nd:YAG: a clinical pilot study. J Dtsch Dermatol Ges 2014;12:322-329.

5 Carney C, Cantrell W, Warner J, Elewski B: Treatment of onychomycosis using a submillisecond 1,064-nm neodymium:yttrium-aluminum-garnet laser. J Am Acad Dermatol 2013;69:578-582.

6 Zhang RN, Wang DK, Zhuo FL, Duan XH, Zhang XY, Zhao JY: Long-pulse Nd:YAG 1,064-nm laser treatment for onychomycosis. Chin Med J (Engl) 2012;125:3288-3291.
Hees H, Raulin C, Bäumler W: Laser treatment of onychomycosis: an in vitro pilot study. J Dtsch Dermatol Ges 2012;10:913918

8 Paasch U, Mock A, Grunewald S, Bodendorf MO, Kendler M, Seitz AT, Simon JC, Nenoff $\mathrm{P}$ : Antifungal efficacy of lasers against dermatophytes and yeasts in vitro. Int J Hyperthermia 2013;29:544-550.

9 Crawford F, Hollis S: Topical treatments for fungal infections of the skin and nails of the foot. Cochrane Database Syst Rev 2007;3: CD001434. 